\title{
Sensor-integrating gears: wear detection by in-situ MEMS acceleration sensors
}

\author{
Julian Peters ${ }^{1} \cdot$ Lorenz Ott $^{1} \cdot$ Matthias Dörr ${ }^{1} \cdot$ Thomas Gwosch $^{1} \cdot$ Sven Matthiesen ${ }^{1}$ iD
}

Received: 29 March 2021 / Accepted: 24 November 2021 / Published online: 23 December 2021

(C) The Author(s) 2021

\begin{abstract}
Gear tooth wear is a common phenomenon leading to malfunctions in machines. To detect wear and faults, gear condition monitoring by vibration is established. The problem is that the measurement data quality for detection of wear by vibration is not good enough with currently established measurement methods, caused by long signal paths of the commonly used housing mounted sensors. In-situ sensors directly at the gear achieve better data quality, but are not yet proved in wear detection. Further it is unknown what analysis methods are suited for in-situ sensor data. Existing gear condition metrics are mainly focused on localized gear tooth faults, and do not estimate wear related values. This contribution aims to improve wear detection by investigating in-situ sensors and advance gear condition metrics. Using a gear test rig to conduct an end of life test, the wear detection ability of an in-situ sensor system and reference sensors on the bearing block are compared through standard gear condition metrics. Furthermore, a machine-learned regression model is developed that maps multiple features related to gear dynamics to the gear mass loss. The standard gear metrics used on the in-situ sensor data are able to detect wear, but not significantly better compared to the other sensors. The regression model is able to estimate the actual wear with a high accuracy. Providing a wear related output improves the wear detection by better interpretability.
\end{abstract}

\section{Sensorintegrierende Zahnräder: Verschleißdetektion durch In-situ MEMS Beschleunigungssensoren}

\section{Zusammenfassung}

Der Verschleiß von Zahnrädern ist ein häufiges Phänomen, das zu Fehlfunktionen in Maschinen führt. Zur Erkennung von Verschleiß und Fehlfunktionen ist die vibrationsbasierte Überwachung des Getriebezustands etabliert. Das Problem ist, dass die Messdatenqualität für die vibrationsbasierte Erkennung von Verschleiß mit den derzeit etablierten Messmethoden nicht gut genug ist, verursacht durch lange Signalwege der üblicherweise verwendeten gehäusemontierten Sensoren. In-situ-Sensoren direkt am Getriebe erreichen zwar eine bessere Datenqualität, haben sich aber in der Verschleißerkennung noch nicht bewährt. Außerdem ist nicht bekannt, welche Analysemethoden für In-situ-Sensordaten geeignet sind. Bestehende Getriebezustandsmetriken konzentrieren sich hauptsächlich auf lokale Zahnradschäden und berechnen keine Werte mit direktem Verschleißbezug. Dieser Beitrag zielt darauf ab, die Verschleißerkennung durch die Untersuchung von In-situ-Sensoren und erweiterten Getriebezustandsmetriken zu verbessern. Unter Verwendung eines Getriebeprüfstands zur Durchführung eines Lebensdauertests werden die Verschleißerkennungsfähigkeiten eines In-situ-Sensorsystems mit denen von Referenzsensoren am Lagerblock verglichen. Darüber hinaus wird ein maschinell erlerntes Regressionsmodell entwickelt, das mehrere Merkmale der Getriebevibrationen auf den Massenverlust des Getriebes abbildet. Die auf die In-situ-Sensordaten angewandten Standard-Getriebezustandsmetriken sind in der Lage, Verschleiß zu erkennen, allerdings nicht wesentlich besser als die Referenzsensoren. Das Regressionsmodell ist in der Lage, den tatsächlichen Verschleiß mit einer hohen Genauigkeit zu schätzen. Die Bereitstellung einer verschleißbezogenen Ausgabe verbessert die Verschleißerkennung durch bessere Interpretierbarkeit.

Sven Matthiesen

sven.matthiesen@kit.edu

$1 \quad$ IPEK-Institute of Product Engineering, Karlsruhe Institute

of Technology (KIT), Karlsruhe, Germany 


\section{Introduction}

Gear tooth wear is one of the major failure modes of gears [1]. The progress of wear on gears results in dynamic transmission error, power transmission losses and high vibration noise levels [2]. Wear in a critical state can also induce faults like teeth breakage, which can cause whole machines to break down. To reduce costs associated with machine breakdowns gear condition monitoring $(\mathrm{CM})$ has been established using various sensors to measure and interpret the gears state [3]. With these techniques, maintenance operations can be scheduled which is also called predictive maintenance. For this purpose, it is essential to estimate a remaining lifetime of the gears using the sensor data. Therefore, the measurements must allow to track the progression of the gear's condition. Besides the detection of particles in lubrication oil [4], a common way to conduct $\mathrm{CM}$ is the use of piezo accelerometers mounted on gearbox housings to measure vibration, which will be focused in this contribution $[3,5,6]$. The measurement data can be analyzed using existing gear condition metrics which output values that are sensitive to faults or wear of the teeth $[7,8]$.

The problem is that the data quality for detection of wear by vibration is not good enough with currently established measurement methods. The wear detection is mainly determined by the measurement data quality and the analyzation methods. Both aspects will be discussed in detail in the following.

\subsection{State-of-the-art}

Data quality is lower for housing mounted accelerometers due to noise and signal transformations occurring in the long signal paths from the source of wear to the sensor which leads to uncertainties in the data interpretation [5, 9-12]. Attempts trying to mount sensors closer to the source of wear to increase data quality and reduce filtering effort exist. An overview is published in [13], the most relevant are presented in the following:

Lewicki et al. [14] mounted micro electro mechanical systems (MEMS) acceleration sensors on a helicopter gear shaft to conduct in-situ measurements. The sensors could detect the gear fault, but only with a short warning time. The focus was on detecting gear faults and not gear wear. Furthermore, the sensors were not mounted directly on the gear wheels, but on the shaft. This effects in longer signal paths resulting in higher uncertainties.

Utakapan et al. [15] mounted piezo accelerometers directly on the gear wheels to improve signal quality. Their goal was to evaluate and optimize gear vibrations and noise, no wear evaluation was performed.
Smith et al. [16] mounted sensors on the planet carrier but received inferior performance compared to exterior sensors, probably due to measurement problems.

Peters et al. [17] presented a sensor-integrating gear wheel where MEMS accelerometers were mounted directly on the gear and a microcontroller for data acquisition mounted on the rotating shaft. MEMS accelerometers were chosen over piezos because they are more compact. The system was able to measure dynamic gear characteristics important for wear detection and showing better signal to noise ratios than bearing block mounted sensors. However, it still needs to be proven that this in-situ MEMS measurement system can detect wear.

To enable wear detection, features that correlate with progressing wear have to be extracted from the vibration time signal, which has been shown by e.g. Bartelmus [18]. However, most gear condition metrics focus on localized fault detection $[7,8]$. The usage of vibration analysis for gear wear detection is not well established, since the correlation between vibration signals and wear are complex due to nonlinearities and random variations [1]. Further, the output values do not have a wear related meaning and need to be analyzed relatively to a healthy state of the gear. This makes it hard to interpret the output values in significance of the wear and estimate the remaining lifetime.

In the following there is an excerpt of common metrics that are sensitive to wear. The root mean square (RMS) indicates the general condition of the gearbox in later stages of degradation. It is sensitive to gearbox load and speed changes. The formula is

$R M S=\sqrt{\frac{1}{N}\left[\sum_{i=1}^{N} S_{i}^{2}\right]}$

with $S=$ vibration signal, $i=$ data point number in vibration signal, $N=$ total number of data points in vibration signal $[7,8]$. The energy ratio (ER) indicates heavy wear, where multiple teeth on the gear are damaged. The formula is

$E R(x)=\frac{\sigma(d)}{\sigma(R)}$

with $\sigma=$ standard deviation, $d=$ difference signal which contains only the gear mesh frequency (GMF) and its harmonics of the gearset under investigation (in a multi-stage gearbox), and $R=$ regular signal which contains primary (shaft) frequency and GMFs with their harmonics of the gearsets except the one under investigation [7, 8].

Machine-learning (ML) models are being researched for damage detection on gears [19]. The focus is on fault detection, not progressing wear. ML models need feature vectors as input which are extracted from the vibration data and can also be the formerly introduced gear metrics. Important steps in application of ML models is dimensionality reduc- 
Table 1 Bearing Block (BB) Sensor Specifications

\begin{tabular}{lll}
\hline & MEMS In-Situ \& BB & Piezo BB \\
\hline Name & IIS3DWB & PCB-356A02 \\
Type & Capacitive MEMS & Piezo \\
Measurement range & $\pm 16 \mathrm{~g}$ & $\pm 500 \mathrm{~g}( \pm 50 \mathrm{~g}$ with amplification of 10) \\
Bandwidth & $6.3 \mathrm{kHz}$ & $5 \mathrm{kHz}$ \\
Resonance frequency & $6.9 \mathrm{kHz}$ & $25 \mathrm{kHz}$ \\
Sensitivity & $0.488 \mathrm{mg} / \mathrm{LSB}$ & $10 \mathrm{mV} / \mathrm{g}(100 \mathrm{mV} / \mathrm{g}$ with amplification-0.0015 mg/LSB with $16 \mathrm{bit}$ ADC at \\
& (Least Significant Bit) & $10 \mathrm{~V}$ range $)$ \\
\hline
\end{tabular}

tion of the feature vectors and the modelling itself. Current research differentiates mostly in these points. Wang et al. [20] found that the feature RMS of the residual acceleration signal is useful in describing gear degradation trends (wear) and used that with a support vector data description model to form a health indicator.

Also, other reduction methods and models are used like empirical mode decomposition or hidden markov models [19]. However, the models used are very specific and highly adapted to the cases of investigation and only few features of the vibration signal are taken into consideration. Most of these models rely on anomaly detection from a model trained on a healthy state and not a continuous tracking of the wear state. Furthermore, they do not correlate the features to an actual wear variable like mass loss.

\subsection{Aim and research questions}

Addressing the problems signal quality and vibration analysis, the contribution aims to improve wear detection by using in-situ sensors and by advancing already existing gear condition metrics for in-situ measurement. The work is structured by the following research questions (RQ):

- RQ1: Can existing gear condition metrics be used with the in-situ MEMS accelerometers to detect wear and does

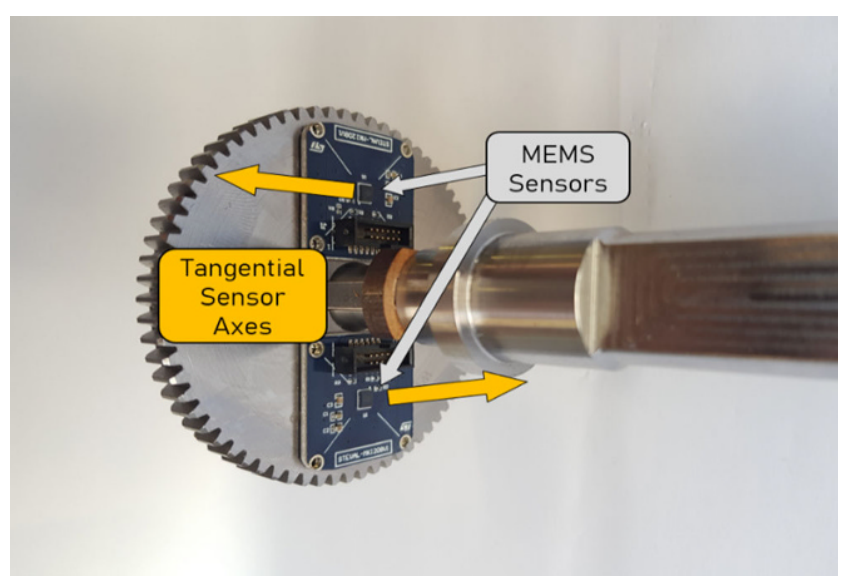

Fig. 1 MEMS Sensors on Gear the higher signal quality of the in-situ measurements improve wear detection?

- RQ2: Can analysis methods for wear detection be improved by using a combination of multiple gear dynamics related features and by estimating a correlation between those features and the actual wear?

In this contribution we aim for a data-driven approach to gain an insight into the gearset, focusing the signal analysis for our in-situ sensor in comparison to external sensors. The goal is to tackle the task of damage recognition with existing gear condition metrics and wear progression related improvements without a detailed analysis of the phenomena and gear specifics.

\section{Materials and methods}

\subsection{Gear test rig with measurement system}

A gear test rig with both in-situ sensors and reference sensors on the housing is used. A more detailed description of the in-situ measurement system can be found in [17]. It consists of two MEMS acceleration sensors (IIS3DWB, ST microelectronics, Geneva, Switzerland), see Table 1, which are glued on the spur gear to measure tangential accelera-

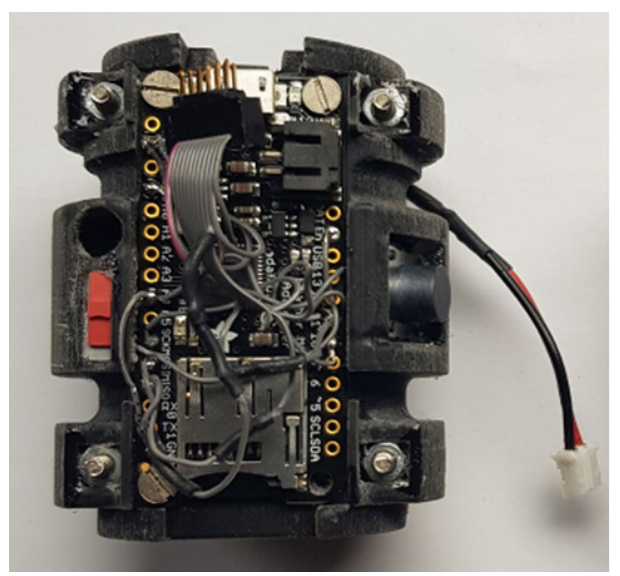

Fig. 2 Microcontroller Board and Battery 
Fig. 3 Gear Test Rig with Measurement System

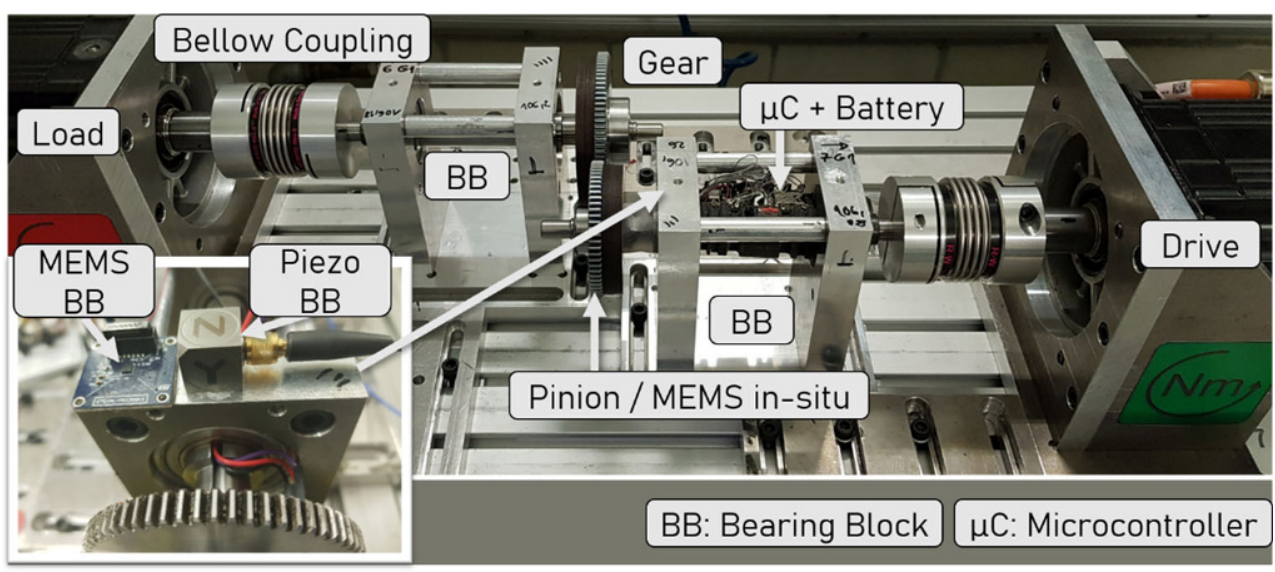

tion (Fig. 1) and a microcontroller development board for data acquisition which is mounted on the gear shaft (Fig. 2).

For reference measurements on the bearing block the sensors from Table 1 were used. First, the IIS3DWB, which is the same one as on the gear to enable a direct comparison between in-situ and bearing block positions (Fig. 3: MEMS BB). Second, the piezo accelerometer (PCB-356A02, PCB Piezotronics, Depew, NY, USA) to ensure a measurement compatible with the state of the art of CM (Fig. 3: Piezo BB).

A single gear stage was used in the test rig (Table 2). The gears are lubricated by grease. There is a demountable splash shield surrounding the gears. As drive and load electric motors (Table 3) are used. The gear test rig with measurement system is depicted in Fig. 3.

\subsection{Data acquisition and postprocessing}

All sensors were sampled with $10 \mathrm{kHz}$. The MEMS sensors were sampled in buffers for $0.68 \mathrm{~s} 44$ times every hour. At a rotational speed of $800 \mathrm{rpm}, 9$ complete rotations of the pinion wheel are covered in one buffer. The piezo sensor was sampled continuously. For comparison to the MEMS sensors the same time windows were extracted. Postprocessing was done with a 5th order butterworth filter, the cutoff frequencies were $5 \mathrm{~Hz}$ (high-pass) and $4000 \mathrm{~Hz}$ (lowpass). This allows the shaft rotation frequency $(13.3 \mathrm{~Hz})$ and the gear mesh frequencies (GMF) up to the 3rd order

Table 2 Characteristics of Gear Wheels

\begin{tabular}{lll}
\hline & Pinion & Gear \\
\hline Number of Teeth & 70 & 90 \\
Module & 1 & 1 \\
Material & C45 milled & C45 milled \\
Face width [mm] & 6.5 & 6.5 \\
Pressure angle $\left[{ }^{\circ}\right]$ & 20 & 20 \\
Max. load momentum $[\mathrm{Nm}]$ & 7.56 & 11.9 \\
Weight [grams] & 219 & 426 \\
\hline
\end{tabular}

$(2800 \mathrm{~Hz})$ to be visible in the spectrum. Shannon's sampling theorem is preserved; the sampling frequency is more than 3 times higher than the maximum frequency of interest.

For evaluating the actual state of wear of the gear three teeth were marked to observe wear patterns and surface roughness optically with a digital microscope (VHX2000, Keyence, Osaka, Japan). Surface roughness has shown to influence vibrations [21]. As an operational variable for the wear the mass loss is used, which has been used successfully in other studies as well. E.g. [22] weighted gear wheels with a scale, or [1] used accumulated mass of particles in oil. We measured mass loss of the pinion directly by a calibrated scale with a sensitivity of $0.01 \mathrm{~g}$, tracked after runtimes $0 \mathrm{~h}, 6 \mathrm{~h}$ and then after every $10 \mathrm{~h}$. The gear was demounted and cleaned by an alcohol solution before microscope and weight measurements. Before and after remounting of the gear the RMS of time data and the frequency spectrum was checked for signs of change in the test rig.

\subsection{Experiment design}

Load and speed of the test rig was kept constant in two phases according to Table 4. These operation conditions match those of power tools, e.g. drilling machines or jigsaws.

The measurement phase has a lower rotational speed to be able to measure more of the higher harmonic multiples

Table 3 Characteristics of Electric Motors

\begin{tabular}{ll}
\hline & Drive \& Load \\
\hline Nominal/Max. Torque [Nm] & $13.1 / 46.9$ \\
Nominal/Max. Rotational Speed & $4500 / 9000$ \\
[rpm] & $\begin{array}{l}\text { Permanent Magnet Syn- } \\
\text { chronous }\end{array}$ \\
\hline
\end{tabular}


Table 4 Phases of Gear Run

\begin{tabular}{llll}
\hline Phase & $\begin{array}{l}\text { Pinion rotational } \\
\text { speed }[\mathrm{rpm}]\end{array}$ & $\begin{array}{l}\text { Gear load } \\
{[\mathrm{Nm}]}\end{array}$ & $\begin{array}{l}\text { Duration } \\
{[\mathrm{min}]}\end{array}$ \\
\hline Measurement & 800 & 9 & 5 \\
Wear progression & 1200 & 12 & 55 \\
\hline
\end{tabular}

of the gear-mesh frequency and to reduce the amplitudes of the acceleration to be within the range of the in-situ MEMS sensor.

The load is 1.2 times higher than the rated load of the pinion (Table 3). This is a common procedure to accelerate the progression of wear and also used e.g. in [3]. The gearset was run until the wear was critical and impaired the function, which means that the teeth were worn off. This moment was tracked by the deviation of the rotational speed control circuit of the drive motor.

\subsection{Wear detection by state-of-the-art gear metrics (RQ1)}

First, the time and frequency data were investigated to ensure a correct measurement by checking the appearance of gear dynamics features (GMFs, sidebands).
Second, the metrics introduced in Chap. 1.1 were used for wear detection. For all sensors, each metric is calculated for each of the sampled buffers every hour (see Chap. 2.2). The values were plotted over the complete runtime. The buffers of every hour are combined via a boxplot, resulting in one box for each hour showing median, quartiles, whiskers and outliers. One plot is created for each metric, combining all sensors and the mass loss as wear progression, which allows a comparison of turning points and gradients to assess the wear detection capability. The metrics and wear are scaled for easier comparison.

\subsection{Wear detection by regression-based machine learning algorithm (RQ2)}

A machine learning model is developed (Fig. 4) that uses regression analysis to infer a relationship between features extracted from measured vibrations and the progression of wear, thus advancing formerly introduced gear metrics by another step.

For the model, the state of gear wear is used as dependent variable and approximated by the mass loss of the gear. The independent variables are features extracted from the measured vibrations.
Data Acquisition
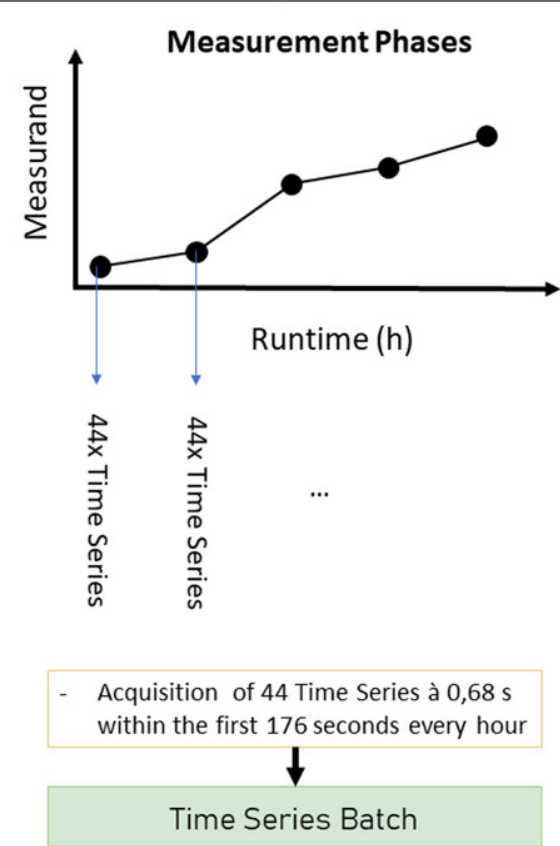

Time Series Batch
Data Processing

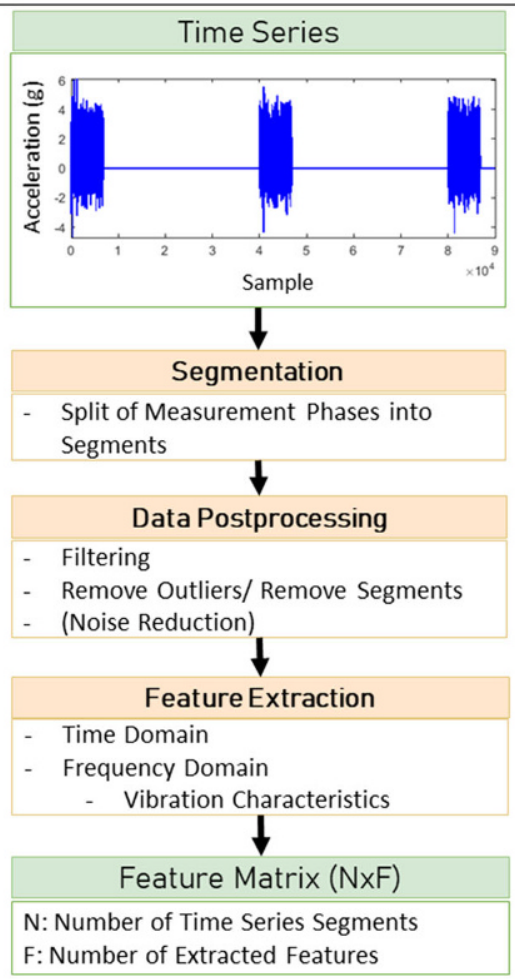

$\mathrm{F}$ : Number of Extracted Features
Data Usage

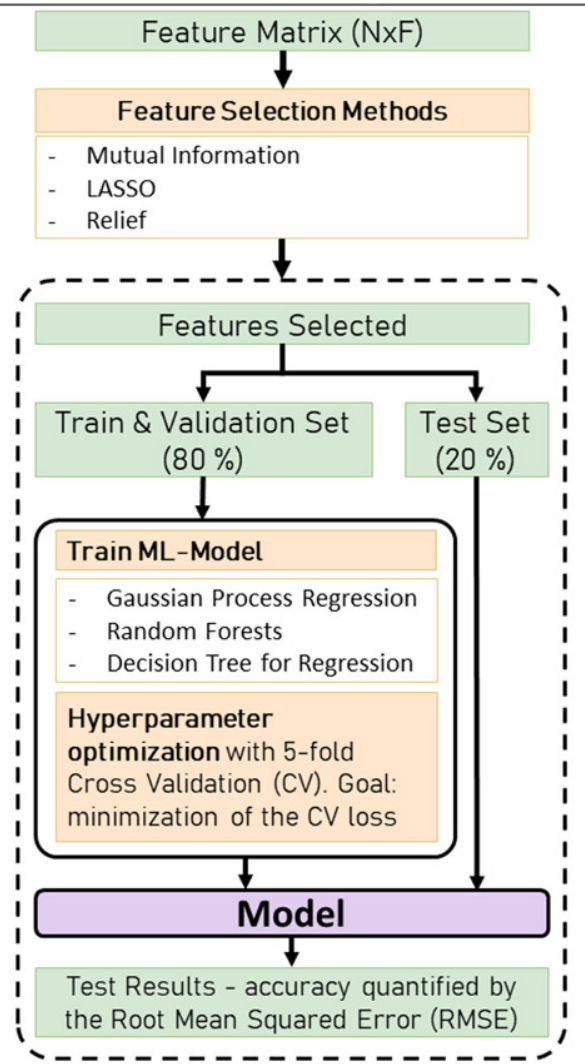

Repetition with different feature selection methods and ML-models

Fig. 4 Machine-Learning Framework 
Building a framework for the regression model includes the steps of data acquisition, data processing and data analysis, which is depicted in Fig. 4.

\section{Results}

\subsection{Actual gear wear progression}

The gears lasted $23 \mathrm{~h}$ of operation until loss of function. Fig. 5 shows the degradation of a tooth profile from 3.7 to $2.78 \mathrm{~mm}^{2}$.

For assessment of actual wear in between, Fig. 6 shows the change of one tooth flank at the specified hours of runtime. The double scalloped wear pattern is visible. Using the definitions from ISO 10825 the teeth show abrasive and rolling wear [23]. Rolling wear is a sign of overloading, which was done on purpose in our tests to achieve a faster wear procedure.

Fig. 7 depicts the surface roughness $(\mathrm{Sa})$ and the mass loss. Sa shows a decreasing trend in 3 phases, indicating a change in the tooth faces. The mass loss shows an exponential growth up to $1.28 \mathrm{~g}$ at its top $(23 \mathrm{~h})$. These parameters have been used successfully in other studies as well for wear assessment (Chap. 2.2). In summary, the ob- servations show that wear occurred and enable the further investigations.

\subsection{Sensor data frequency spectrums}

In general: The frequency spectrums of all the sensor signal data (Figs. 8, 9 and 10) show the GMFs. MEMS insitu, MEMS bearing block and Piezo bearing block data clearly show the first two harmonic multiples at 933.3 and $1866.7 \mathrm{~Hz}$ as well as two sets of sidebands to each side. The $3 \mathrm{rd}$ harmonic at $2800 \mathrm{~Hz}$ is also visible, however barely distinguishable from noise. An upwards trend of the peaks of the GMFs with proceeding runtime is observable in all sensor data, which is a good indication for the wear detectability carried out afterwards.

Also, the sensor data directly before and after remounting for wear assessment of the gears was observed for signs of change. It is visible that no additional frequencies occurred that were not there before. The change in gear mesh and sideband amplitudes of MEMS bearing block was less than $20 \%$, which is in the same range or less as the hourly changes without remounting procedure. The change due to wear is significantly higher with more than $100 \%$. Also, the overall change in frequency spectrum amplitudes was
Fig. 5 Tooth profile and area at specified runtimes
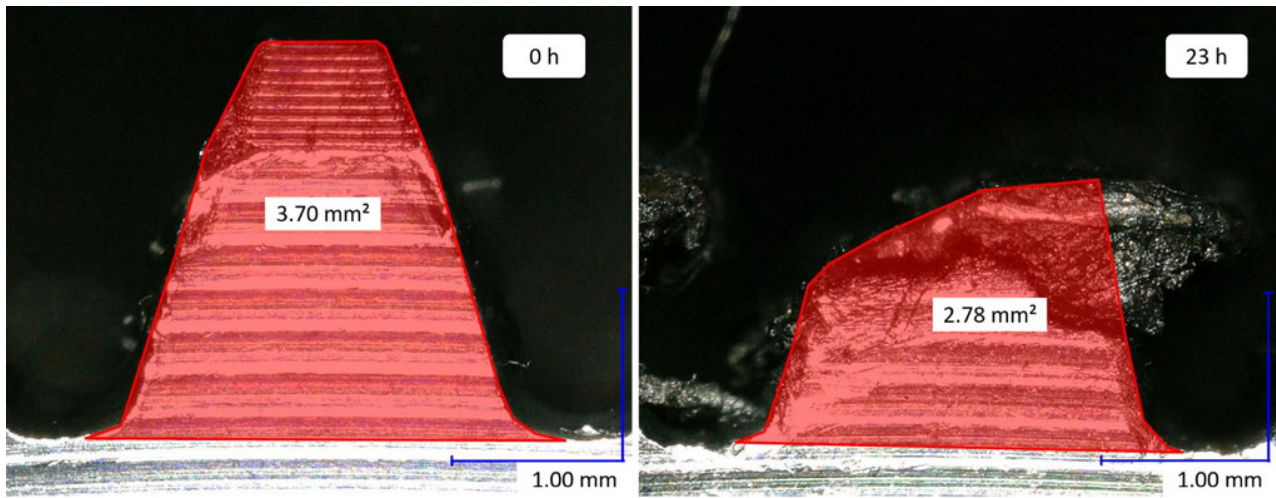

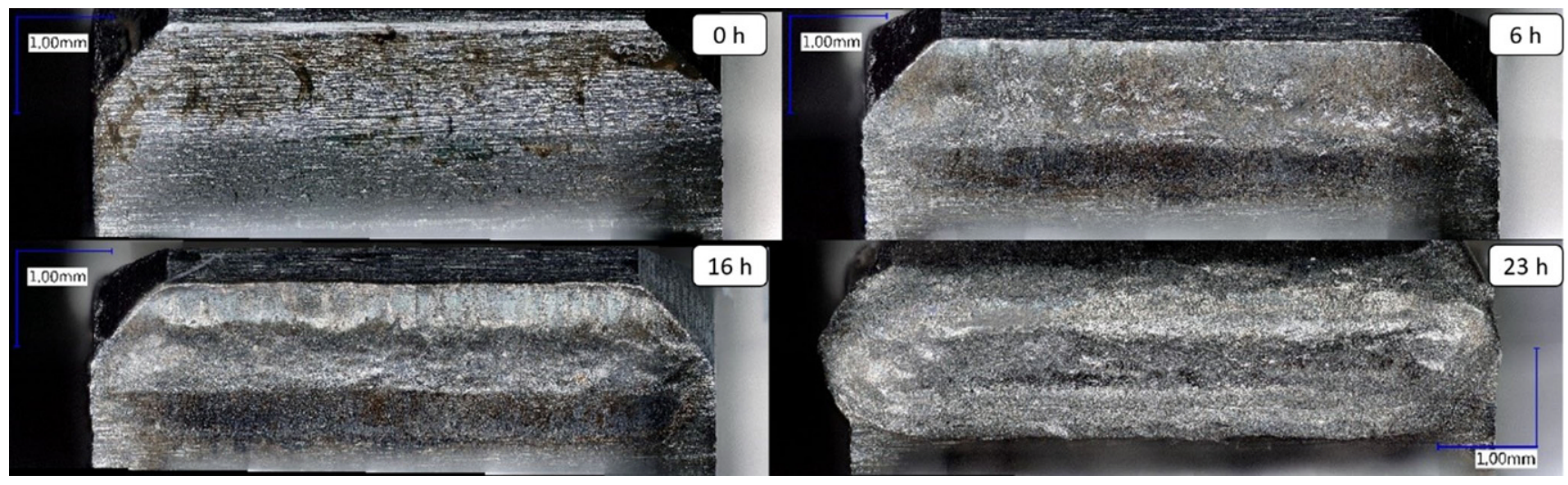

Fig. 6 Gear Tooth Flank at specified Runtimes 
Fig. 7 Surface Roughness Sa and Mass Loss

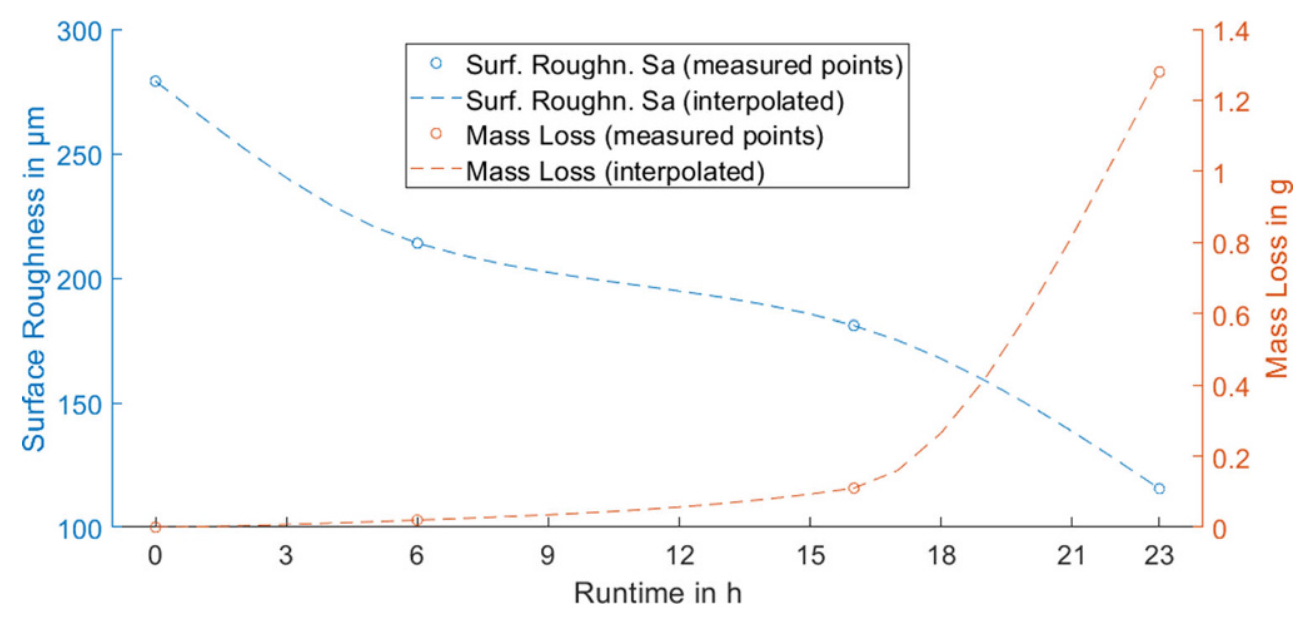

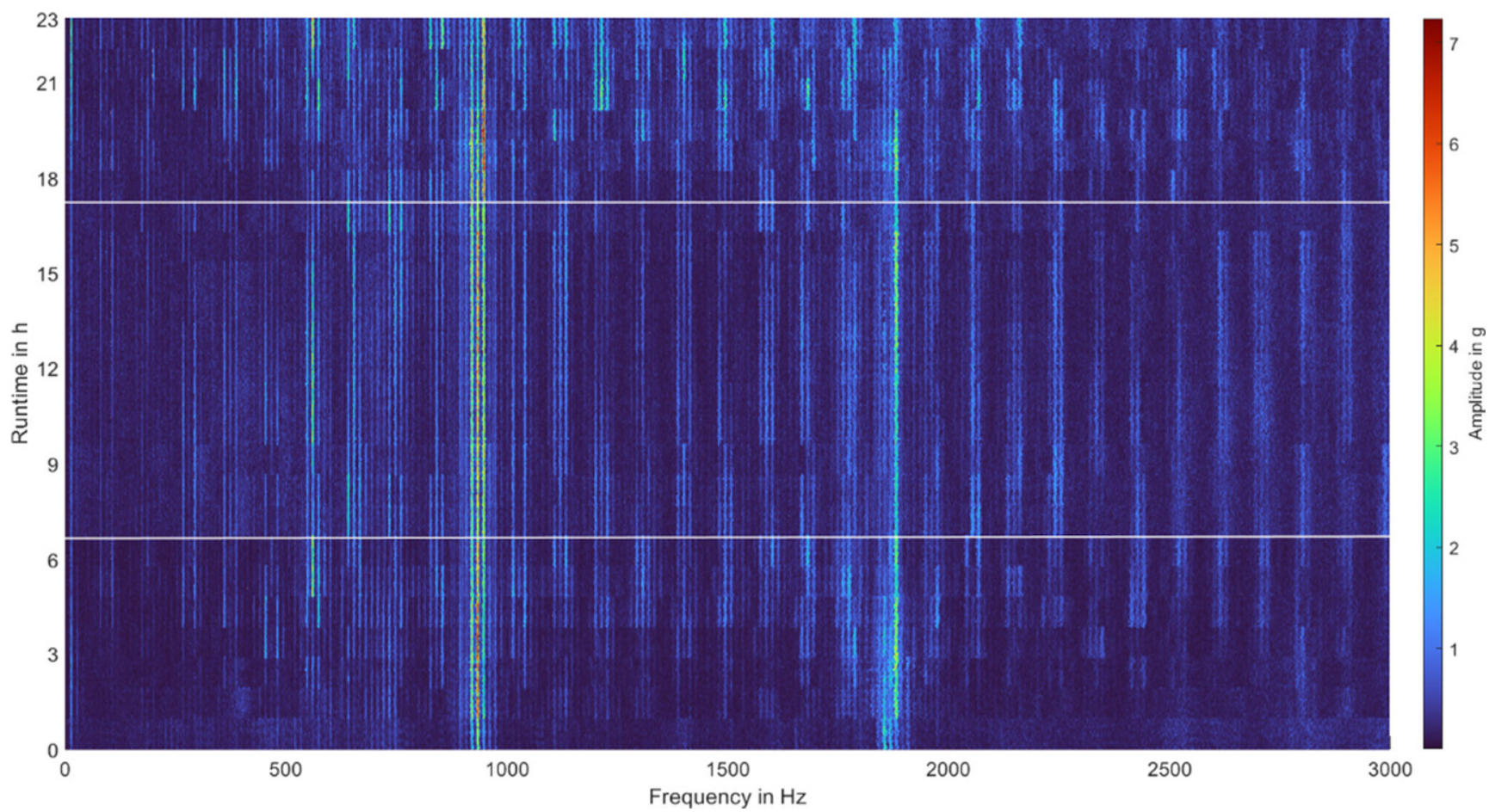

Fig. 8 Acceleration Frequency Spectrum: In-Situ MEMS Sensor, horizontal lines indicate test rig halts for wear assessments

in the same range or less as the hourly changes without the remounting procedure.

The in-situ MEMS sensor reached the limit of its measurement range a few times and presumably exceeded it which may have an impact on the data. Furthermore, the data of the piezo sensor on the bearing block could not be recorded between runtimes 9 and $16 \mathrm{~h}$ due to a memory overflow.

MEMS in-situ (Fig. 8) vs. MEMS bearing block (Fig. 9): The peaks of the GMFs of the in-situ data are higher than those of the bearing block. The upwards trend at the end representing the wear progression is steeper in the bearing block sensor data. In-situ from runtimes $18 \mathrm{~h}$ and later the $1 \mathrm{st}$
GMF reduces whereby the 1 st sidebands at $933.3 \pm 13.3 \mathrm{~Hz}$ increase rapidly, especially the higher one. This is also earlier visible at the 2nd GMF and can be regarded as an indicator of wear. The bearing block sensor does not show the 1 st sidebands but the $2 \mathrm{nd}$ ones at $933.3 \pm 2 * 13.3 \mathrm{~Hz}$ which also increase in amplitude.

The in-situ shows the gear rotation frequency $(13.3 \mathrm{~Hz})$ and lots of peaks at integer multiples of it with the GMFsideband structure. The same is observable in the bearing block sensor data with lower amplitudes, however not at the first order.

Bearing block MEMS (Fig. 9) vs. piezo (Fig. 10): Both bearing block sensors show similar frequencies. The piezo sensor 


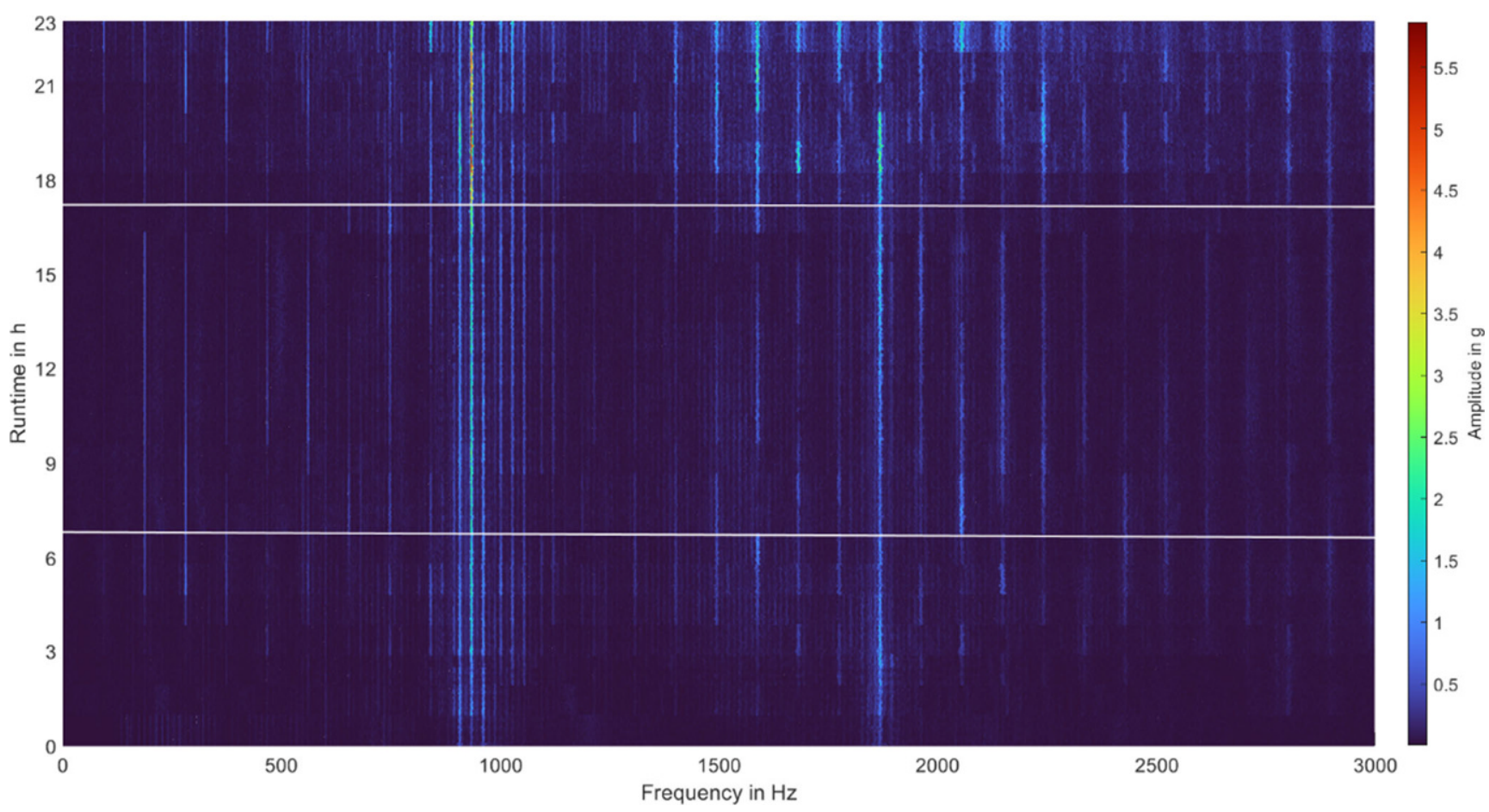

Fig. 9 Acceleration Frequency Spectrum: Bearing Block MEMS Sensor Z, horizontal lines indicate test rig halts for wear assessments

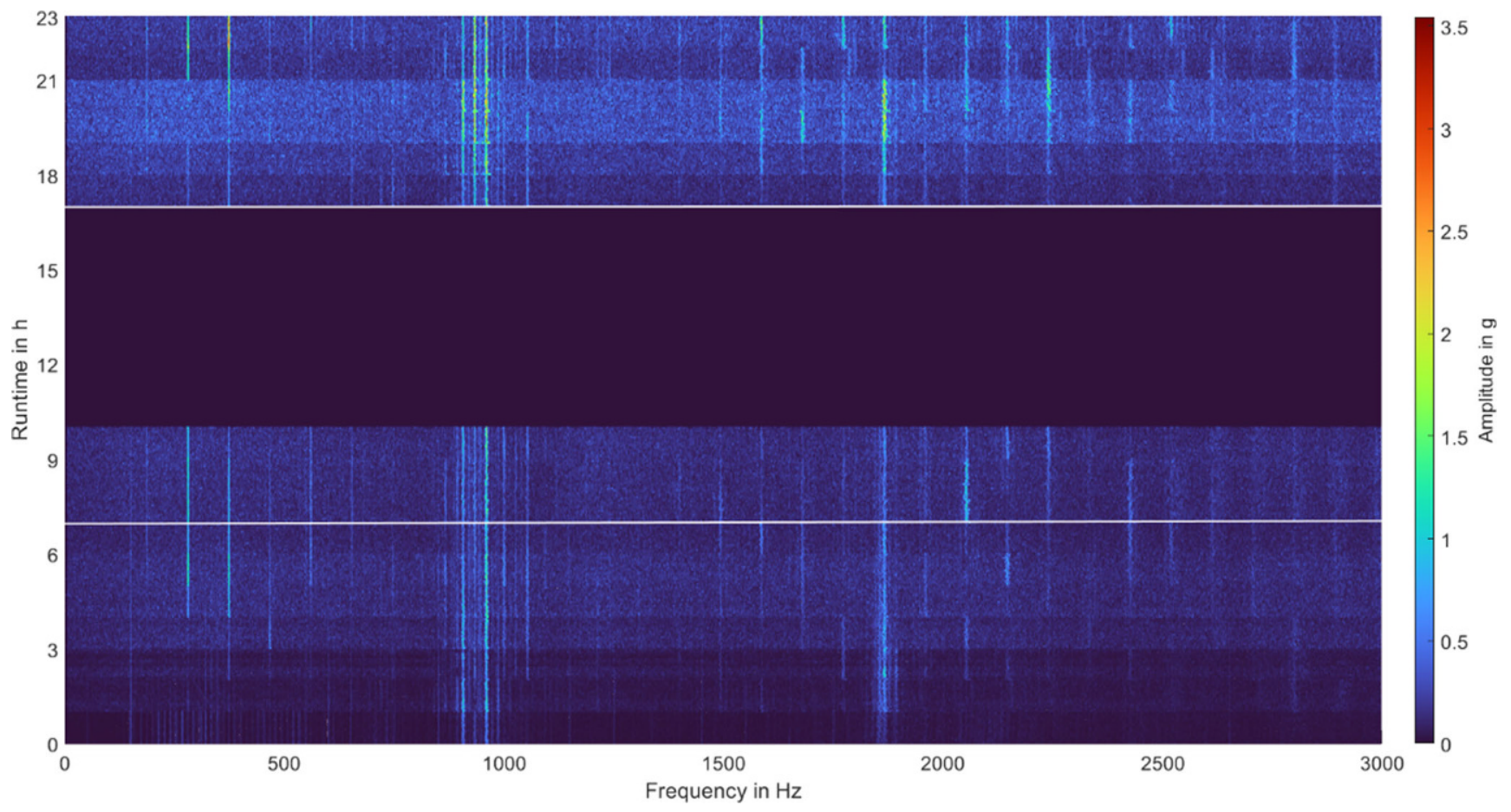

Fig. 10 Acceleration Frequency Spectrum: Bearing Block Piezo Sensor Z, horizontal lines indicate test rig halts for wear assessments 
Fig. 11 RMS of Sensor Data with Vertical Lines at Run-In and Accelerated Wear Phase

Fig. 12 Energy Ratio of Sensors with Vertical Lines at Run-In and Accelerated Wear Phase
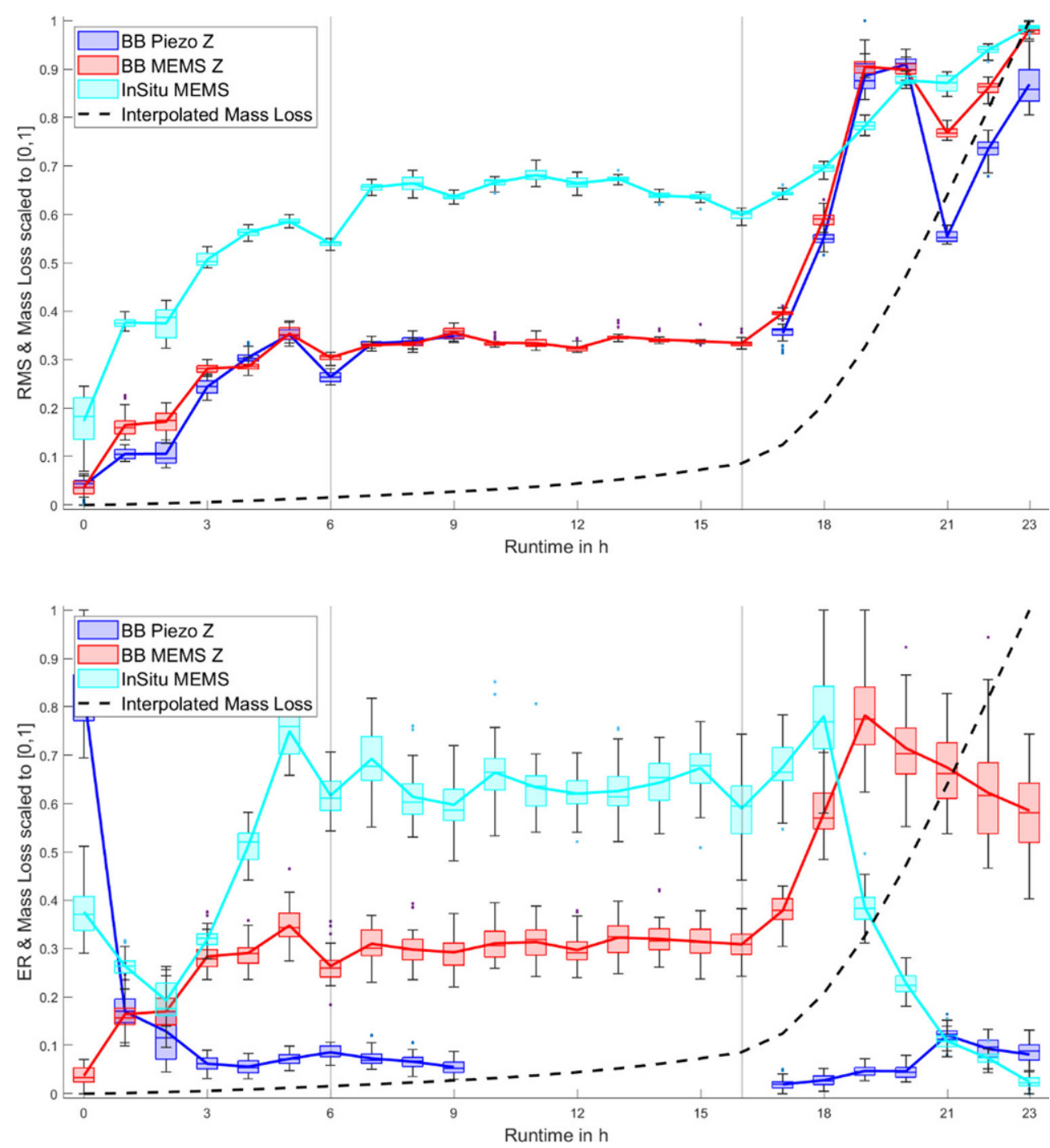

data has lower amplitudes and more noise than the MEMS. The upwards trend in the 1st GMF is similar, in the 2nd GMF is steeper in the piezo sensor data.

\subsection{Wear detection by state-of-the-art gear metrics (RQ1)}

The gear condition metrics RMS (Eq. 1) and ER (Eq. 2) for in-situ and bearing block acceleration signals are shown in the following figures. For comparing the results, the quality is assessed by comparing the turning points and gradients in three phases (run-in, stationary condition, accelerated wear) which are marked in the figures.

In the first phase the RMS (Fig. 11) shows the run-in period $(6 \mathrm{~h})$ of the gear pair by an increase in all sensors. The in-situ sensor yields higher values in stationary phase. All sensors indicate the start point of accelerated wear at about the same time (16h). In the last phase the gradient of the in-situ sensor is lower compared to the mass loss, whereas the bearing block sensors gradient is higher with a break at $21 \mathrm{~h}$. The MEMS in-situ does not have this break, but its range was exceeded sometimes in the last phase. Bearing block MEMS and piezo match well apart from a slight deviation at the end. The change of RMS absolute value in bearing block MEMS from before to after the remountings $(6 \mathrm{~h}, 16 \mathrm{~h})$ is $<5 \%$ whereas the overall change due to wear is $>400 \%$.

The ER (Fig. 12) shows a very fluctuating process for all sensor data. In the run-in period the MEMS in-situ and bearing block sensors show overall increasing values, followed by a stationary phase until the accelerated wear phase at $16 \mathrm{~h}$. The gradient of the wear in the last phase is best covered by the bearing block MEMS sensor, followed by the in-situ MEMS sensor. Contrary to the wear, the in-situ MEMS decreases again after $18 \mathrm{~h}$ and continues falling until the end. The MEMS bearing block sensor covers the increase longer until $19 \mathrm{~h}$, but also starts falling before the end. The bearing block piezo sensor shows a bathtub curve, 
Fig. 13 Estimation of Wear (Mass Loss)—Regression Model Output

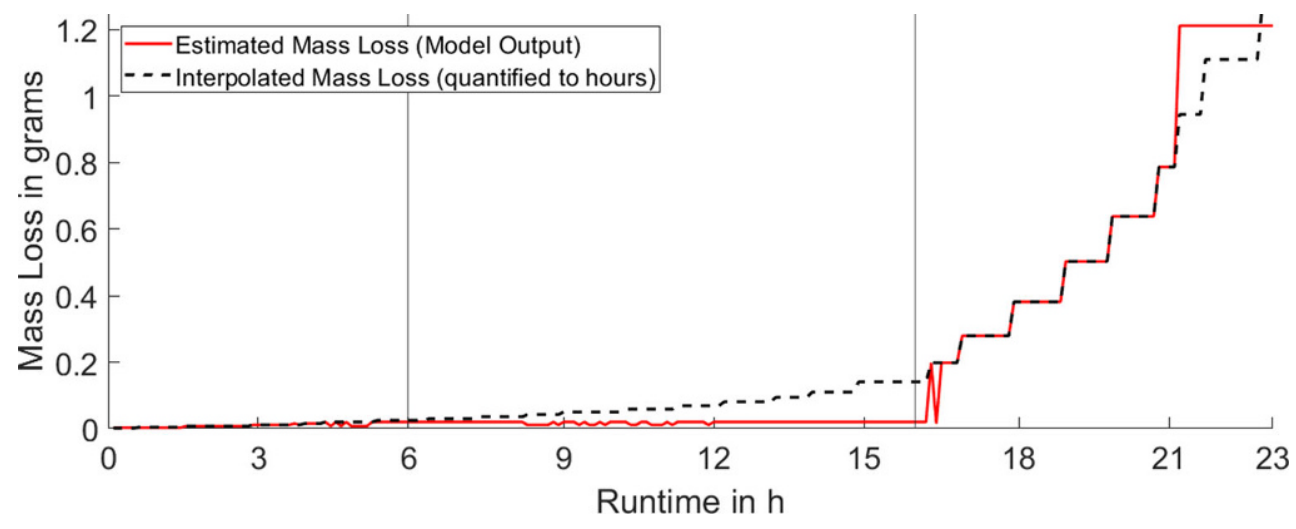

which is a common characteristic of the gear life, with a decrease in the run-in phase, followed by a stationary phase and a slight increase in the accelerated wear phase. It also starts to decrease a little before the end.

\subsection{Regression-based machine learning algorithm (RQ2)}

The model provides the best results with decision tree as model and mutual information as feature selector with a RMSE of $0.028 \mathrm{~g}\left(\mathrm{RRMSE}=2.2 \%, \mathrm{R}^{2}=99.48 \%\right)$. The output of the model, the estimated mass loss, is shown in Fig. 13 together with the interpolated mass loss which is quantified hourly to fit the measurement periods every hour (Chap. 2.3). From the beginning up to the point of accelerated wear $(16 \mathrm{~h})$ the estimation does not fit the slight increase of the wear. But afterwards the estimation matches the wear very well apart from the last two hours, where it deviates again in a conservative way.

A selection of the features that have the strongest impact on the quality according to the feature selection algorithm are:

- Peak heights and energies of GMFs (mainly 2nd and 3rd) and their sidebands

- Peak to peak differences in GMFs and sidebands

- RMS

- Percentils 20 and $80 \%$

- Sum of difference signals (gear mesh signal and harmonics)

\section{Discussion}

The results concerning RQ1 show that the in-situ sensor system is able to detect wear using the standard gear condition metrics, but not significantly better compared to the other sensors in our case. Especially the MEMS sensor on the bearing block allows the same quality of wear detection and is considerably easier to mount.
Lewicki et al. [14] also states similar results comparing in-situ MEMS and housing mounted sensors with standard metrics like RMS. However, in their case the in-situ performed slightly better and the focus was fault detection, not wear. Another comparison between in-situ and external vibration measurements in the case of planetary gear fault detection was done by Smith et al. [16], receiving inferior performance for the in-situ sensors. However, they stated problems with the in-situ measurements likely for that. The results of our contribution show that in-situ has no inferior performance in the case of single stage gearboxes. For planetary gears this still needs to be investigated.

In a laboratory style single stage gearbox with few sources of noise the in-situ measurement with standard metrics does not seem worth the higher implementation effort. However, in a multi stage gearbox with more sources of noise, advantages of the in-situ measurement are expected: Filtering techniques for bearing block sensors to separate the gear pairs will be necessary, increasing the postprocessing effort and the uncertainty, which the in-situ sensors probably will not need. Also, the results show that the gear rotation frequency is well visible in the spectrum, which opens up possibilities of order tracking, enabling analyzations in varying operation conditions.

Another point is, that the in-situ sensor may not have performed as well as possible, because its measurement range was exceeded several times. For many gear condition metrics, the heights of the amplitudes are important. It is expected, that a sensor with a higher range improves interpretability of the metrics results in-situ. The sensor used in this contribution was chosen because of its high bandwidth and digital output, enabling an integration with microcontrollers. Also, the results of other studies, e.g. [14, 15, 24] did not make believe that the acceleration at the gear wheels would exceed the range of the sensor.

The piezo sensor with sophisticated amplification and measurement system does not provide better, in some cases even inferior wear detection than the MEMS sensor with the microcontroller development board in our test case. This 
refines the findings of Fromberger et al. [6] who stated inferior performance of MEMS accelerometers for some cases.

In the following, limitations are discussed that might influence the results. This study included only one type of gears. Hence, transferability of the proposed in-situ measurement method to other types needs to be shown in future studies. The rapid wear progression after runtime $16 \mathrm{~h}$ could also originate from lubrication issues. For the weight measurement it was necessary to clean and re-lubricate the gear (Sect. 2.2) which may have changed the wear progression. However, this does not change the findings of this contribution, because the origin of the wear is not in focus. Lubrication issues even are a realistic use case in e.g. power tools.

Another point connected with the intermediate weight measurements is the de- and re-mounting of the gear. An influence of the procedure on the test rig cannot be ruled out completely, but it was kept to a minimum by design of the bearings, which did not need to be touched for the re-mounting procedure. Vibration data comparison shows no visible difference in the time domain and only slight changes of the amplitudes of the frequency spectrum which were well below the change due to wear and no new frequencies occurred.

The in-situ sensor shows large 1st order shaft frequency sidebands which are only faintly visible in the bearing block sensors. Lewicki et al. [14] also experienced that, explaining it with the rotating frame of reference of the in-situ sensor. Another possible explanation is a resonance of the sensor system on the rotating part, resulting in higher amplitudes there. This will be investigated more detailed in future studies.

The introduced machine-learned regression model (RQ2) in combination with the in-situ sensor system can improve wear detection by estimating the actual state of wear (gear mass loss). The feature selection algorithm chose many features related to gear dynamics. This supports the state of research, that gear wear correlates to those gear dynamics features which originate from the loaded transmission error [1]. The model takes several of those features into account to estimate the gear wear, thus improving wear detection by single featured state of the art metrics [7, 8]. In fact, some gear metrics even are inputs of the model, the RMS and energy values are within the best features, which agrees with the findings of Wang et al. [20]. In our test case the model shows a very good fit of the actual gear wear in a critical moment when the wear accelerates. Considering an application this would be the time to take further investigations and schedule maintenance. The standard metrics also showed upward trends indicating a change in the wear process to the same time as the regression model. But they do not give a wear related output making it hard to assess the significance of the change of wear which the regression model can provide.

This method advances the analyzation of vibrations by estimating a correlation between the features extracted from the vibration signal and the actual wear (mass loss) in retrospective. Therefore, historic data of a gear test run is needed. Since gears are standardized machine elements and manufacturers anyway need to test run their machines, this initial acquisition of data is not considered to be problematic. Limitations of the model are the gear type and operating conditions like rotation frequency, which influence the input features of the model and may lead to deviations of its output.

\section{Summary and outlook}

In this contribution the wear detection capability of an insitu MEMS acceleration sensor system according to [17] was compared to accelerometers on bearing blocks using standard gear condition metrics. The results show that the in-situ sensor system is able to detect wear using standard gear condition metrics, but not significantly better compared to the other sensors on the bearing block in our single stage test rig.

Furthermore, an advancement of gear condition metrics was presented based on machine-learned regression to estimate the actual state of wear (mass loss) with the in-situ sensor data. This can improve interpretability of the wear detection. In future it is attempted to show transferability to gears of other types. Especially the features selected of the algorithm, if they stay the same or vary, is of interest. Also, an advancement of the model to deal with varying operating conditions and to enable online prediction during runtime is aimed. For that, the rotation frequency shall be used which is measured well by the in-situ sensor.

Acknowledgements The authors thank the Dr.-Ing. Willy Höfler foundation for supporting this contribution.

Funding Open Access funding enabled and organized by Projekt DEAL.

Conflict of interest J. Peters, L. Ott, M. Dörr, T. Gwosch and $\mathrm{S}$. Matthiesen declare that they have no competing interests.

Open Access This article is licensed under a Creative Commons Attribution 4.0 International License, which permits use, sharing, adaptation, distribution and reproduction in any medium or format, as long as you give appropriate credit to the original author(s) and the source, provide a link to the Creative Commons licence, and indicate if changes were made. The images or other third party material in this article are included in the article's Creative Commons licence, unless indicated otherwise in a credit line to the material. If material is not included in the article's Creative Commons licence and your intended use is not permitted by statutory regulation or exceeds the permitted use, you will 
need to obtain permission directly from the copyright holder. To view a copy of this licence, visit http://creativecommons.org/licenses/by/4.0/.

\section{References}

1. Hu C, Smith WA, Randall RB, Peng Z (2016) Development of a gear vibration indicator and its application in gear wear monitoring. Mech Syst Signal Process 76-77:319-336. https://doi.org/ 10.1016/j.ymssp.2016.01.018

2. Wojnarowski J, Onishchenko V (2003) Tooth wear effects on spur gear dynamics. Mech Mach Theory 38(2):161-178. https://doi.org/ 10.1016/S0094-114X(02)00091-5

3. Randall RB (2011) Vibration-based condition monitoring: industrial, automotive and aerospace. John Wiley \& Sons, Chichester. https://doi.org/10.1002/9780470977668

4. Williams JA (2005) Wear and wear particles-some fundamentals. Tribol Int 38(10):863-870. https://doi.org/10.1016/j.triboint.2005. 03.007

5. Martin G, Vogel S, Schirra T, Vorwerk-Handing G, Kirchner E (2018) Methodical evaluation of sensor positions for condition monitoring of gears. In: Proceedings of NordDesign 2018 Linköping, Sweden

6. Fromberger M, Sendlbeck S, Rothemund M, Götz J, Otto M, Stahl K (2019) Comparing data sources for condition monitoring suitability. Forsch Ingenieurwes 83(3):521-527. https://doi.org/10. 1007/s10010-019-00331-y

7. Samuel PD, Pines DJ (2005) A review of vibration-based techniques for helicopter transmission diagnostics. J Sound Vib 282(1-2): 475-508. https://doi.org/10.1016/j.jsv.2004.02.058

8. Večeř P, Kreidl M, Šmíd R (2005) Condition indicators for gearbox condition monitoring systems. Acta Polytech 45(6):35-43

9. Vorwerk-Handing G, Gwosch T, Schork S, Kirchner E, Matthiesen S (2020) Classification and examples of next generation machine elements. Forsch Ingenieurwes 84(1):21-32. https://doi.org/10.1007/ s10010-019-00382-1

10. Kirchner E, Martin G, Vogel S (2018) Sensor integrating machine elements: key to in-situ measurements in mechanical engineering. In: $23^{\circ}$ Seminário Internacional de Alta Tecnologia

11. Hausmann M, Koch Y, Kirchner E (2021) Managing the uncertainty in data-acquisition by in situ measurements: a review and evaluation of sensing machine element-approaches in the context of digital twins. IJPLM 13(1):48. https://doi.org/10.1504/IJPLM. 2021.115700

12. Welzbacher P, Schulte F, Neu M, Koch Y, Kirchner E (2021) An approach for the quantitative description of uncertainty to support robust design in sensing technology. Des Sci 7:e18. https://doi.org/ 10.1017/dsj.2021.19

13. Peters J, Ott L, Gwosch T, Matthiesen S (2020) Requirements for sensor integrating machine elements: a review of wear and vibration characteristics of gears. KIT Scientific Working Papers, vol 157. Karlsruher Institut für Technologie (KIT), Karlsruhe https://doi.org/ 10.5445/IR/1000127139

14. Lewicki D, Lambert N, Wagoner R (2015) Evaluation of MEMSbased wireless accelerometer sensors in detecting gear tooth faults in helicopter transmissions. NASA, Cleveland

15. Utakapan T, Kohn B, Fromberger M, Otto M, Stahl K (2017) Evaluation of gear noise behaviour with application force level. Forsch Ingenieurwes 81(2-3):59-64. https://doi.org/10.1007/s10010-0170228-y

16. Smith W, Deshpande L, Randall R, Li H (2013) Gear diagnostics in a planetary gearbox: a study using internal and external vibration signals. Int J Cond Monitor 3(2):36-41. https://doi.org/10. 1784/204764213808146617

17. Peters J, Ott L, Dörr M, Gwosch T, Matthiesen S (2021) Design of sensor integrating gears: methodical development, integration and verification of an in-Situ MEMS sensor system. Procedia Cirp 100:672-677. https://doi.org/10.1016/j.procir.2021.05.142

18. Bartelmus W (2003) Diagnostic information on gearbox condition for mechatronic systems. Trans Inst Meas Control 25(5):451-465. https://doi.org/10.1191/0142331203tm0099oa

19. Wang D, Tsui K-L, Miao Q (2018) Prognostics and health management: a review of vibration based bearing and gear health indicators. IEEE Access 6:665-676. https://doi.org/10.1109/ACCESS. 2017.2774261

20. Wang D, Tse PW, Guo W, Miao Q (2011) Support vector data description for fusion of multiple health indicators for enhancing gearbox fault diagnosis and prognosis. Meas Sci Technol 22(2):25102. https://doi.org/10.1088/0957-0233/22/2/025102

21. Yang Y, Smith W, Borghesani P, Peng Z, Randall RB (2015) Detecting changes in gear surface roughness using vibration signals. In: Acoustics Hunter Valley, Australia

22. Brandão JA, Cerqueira P, Seabra JH, Castro MJ (2016) Measurement of mean wear coefficient during gear tests under various operating conditions. Tribol Int 102:61-69. https://doi.org/10.1016/j. triboint.2016.05.008

23. International Organization for Standardization (1995) ISO 10825:1995-08, gears-wear and damage to gear teethterminology. Beuth, Berlin

24. Heider MK (2012) Schwingungsverhalten von Zahnradgetrieben: Beurteilung und Optimierung des Schwingungsverhaltens von Stirnrad- und Planetengetrieben, 1st edn. Dr. Hut, München 